\title{
Gasos Guínicos \\ Uso de N-Acetilcisteína tópica en Ictiosis: Experiencia clínica en una serie de casos
}

\author{
Ligia Aranibar ${ }^{1}$, Nadia Vega $^{1}$, Gabriel Neely ${ }^{2}$
}

\section{RESUMEN}

Las ictiosis son trastornos generalizados de la queratinización, que se caracterizan por presentar hiperqueratosis y/o descamación. El tratamiento es sintomático e incluye terapias tópicas y sistémicas. La N-Acetilcisteína (NAG) tópica ha mostrado utilidad en algunos reportes. Se describirá la experiencia con el uso de NAC 10\% y urea 5\% en novobase II (NB II) en 6 pacientes con distintos tipos de ictiosis hereditarias. Caso $\mathrm{N}^{\circ} 1$ : Escolar masculino de 7 años con Ictiosis lamelar (IL) en tratamiento con fórmula de NAC hace 4 años con buena respuesta. Caso N², 3 y 4: Escolar masculino de 7 años, escolar masculino de 5 años y lactante masculino de 1 año 8 meses con Ictiosis recesiva ligada a $\mathrm{X}$ (IRLX), en tratamiento con fórmula de NAC con buena respuesta. Caso N5: Mujer de 18 años con Eritrodermia ictiosiforme congénita (EIC), inicia tratamiento con fórmula de NAC, pero se suspende por mala tolerancia. Caso $N^{\circ} 6$ : Preescolar femenina de 3 años, con Ictiosis epidermolítica (IE), inicia tratamiento con fórmula de NAC con mala tolerancia por lo que también se suspende. Se evaluaron los efectos de la fórmula de NAG en diferentes tipos de ictiosis, mostrando un buen perfil de seguridad y eficacia en IL e IRLX, sin embargo, en EIC e IE su uso estuvo restringido por efectos adversos. El tratamiento con fórmula de NAC presenta buena respuesta y tolerancia en pacientes con IL e IRLX, por lo que podría considerarse en el tratamiento habitual de estos pacientes.

Palabras claves: Ictiosis hereditarias; Queratinización; NAcetilcisteína Tópica; Eficacia.

\section{SUMMARY}

Ichthyosis are generalized disorders of keratinization, characterized by hyperkeratosis and/or scaling. Treatment is symptomatic and includes topical and systemic therapies. Topical N-acetylcysteine (NAG) has shown utility in some reports. We describe the experience using 10\% NAC and 5\% urea in novobase II (NB II) in 6 patients with different types of hereditary ichthyosis. Case $\mathrm{N}^{\circ} 1$ : 7-year-old boy with lamellar Ichthyosis (IL) in treatment with NAC formula 4 years ago, with good response. Case $\mathrm{N}^{\circ} 2,3$ and 4: 7-year-old boy, 5 -year-old boy and 1-year-8-month-old male infant, with $\mathrm{X}$-recessive recessive Ichthyosis (IRLX) in treatment with NAC formula with good response. Case N5: An 18-year-old woman with congenital ichthyosiform erythroderma (EIC) begins treatment with NAC formula but is discontinued because of poor tolerance. Case No. 6:3-year-old girl, with epidermolytic Ichthyosis (IE), starts treatment with NAC formula with poor tolerance and is also suspended. The effects of the NAG formula on different types of ichthyosis were evaluated, showing a good safety and efficacy profile in IL and IRLX, however, in EIC and IE, its use was restricted by adverse effects. Treatment with NAC formula has a good response and tolerance in patients with IL and IRLX, so it could be considered in the usual treatment of these patients.

Key words: Hereditary Ichthyosis; Keratinization; Topical N-Acetylcysteine; Efficacy.

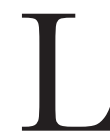
as ictiosis son un grupo heterogéneo de trastornos generalizados de la queratinización, caracterizados por presentar un grado variable de hiperqueratosis y/o descamación de la piel. ${ }^{1,2}$ Las ictiosis hereditarias corresponden a trastornos genéticos, a diferencia

${ }^{1}$ Departamento Dermatología, Universidad de Chile, Santiago, Chile. ${ }^{2}$ Facultad de Medicina, Universidad de Chile, Santiago, Chile

Correspondencia: Nadia Vega. Correo electrónico: almendra.nv5@gmail. com. Celular: +56 9 77370148. Dirección: Santos Dumont 999, Independencia. Código postal: 8380456. de las ictiosis adquiridas que pueden ser secundarias a un diverso grupo de patologías. ${ }^{2}$ Según la nueva clasificación de ictiosis hereditarias (Conferencia de consenso de Sorènze, 2009), basada en características clínicas, moleculares y fisiopatológicas, se distinguen 36 tipos de ictiosis divididas en dos subgrupos: las no sindrómicas, con compromiso exclusivamente cutáneo y las sindrómicas que presentan manifestaciones extracutáneas. ${ }^{1}$ Existen pocos datos sobre la epidemiología de las ictiosis. En Estados Unidos se ha estimado una prevalencia al nacimiento de 1:100.000 para la Ictiosis lamelar (IL) y 1:200.000 para la Eritrodermia Ictiosiforme congénita (EIC). ${ }^{3}$ 
El tratamiento es sintomático e incluye desde terapias tópicas hasta tratamiento con retinoides sistémicos en casos severos o refractarios. ${ }^{3}$ La $\mathrm{N}$-acetilcisteína (NAC) es un agente antioxidante que ha mostrado utilidad como tratamiento tópico en reportes de ictiosis lamelar y se basa en su capacidad de inhibir la proliferación de queratinocitos, sin un efecto citotóxico. ${ }^{4,5}$

Reportamos la experiencia con el uso de NAC al 10\% y urea 5\% en novobase II (NB II) en 6 pacientes con distintos tipos de ictiosis hereditarias.

\section{REPORTE DE GASOS}

Presentamos seis pacientes con distintos tipos de ictiosis (Tabla 1) a los cuales se les indicó receta magistral con los componentes mencionados. Se recomendó su uso posterior al baño y se les indicó a los padres aplicar una película fina sobre la piel afectada. A continuación se describen los casos brevemente:

Paciente $\mathrm{N}^{\circ} 1$ : Escolar masculino de 7 años, con diagnóstico de IL y estudio genético compatible (mutación TGM-1). Nació como bebe colodión de 36 semanas de gestación, evolucionando luego de unas semanas con escamas gruesas y oscuras de distribución generalizada, asociado a ectropión y queratodermia palmoplantar. A los 2 años inicia tratamiento con acitretin $0,5 \mathrm{mg} / \mathrm{kg} /$ día por 6 meses, luego $0,25 \mathrm{mg} / \mathrm{kg} /$ día por 3 meses, revirtiendo el ectropión por lo cual no requirió cirugía reparadora. Desde los 4 años inicia tratamiento con fórmula de NAC cada 12 horas durante un año con buena respuesta y tolerancia desde las 2 semanas de uso. Actualmente, se mantiene en tratamiento con una aplicación diaria. (Figura 1)

Paciente $\mathrm{N}^{\circ}$ 2: Escolar masculino de 7 años, con diagnóstico de Ictiosis recesiva ligada a X (IRLX) sindrómica dado su asociación a criptorquidia. De sus antecedentes perinatales, destaca ser recién nacido de termino de parto prolongado. A los meses de vida, presenta descamación oscura en extremidades y tronco, que respeta cara, pliegues, palmas y plantas. Inicialmente recibe tratamiento con emolientes y queratolíticos con respuesta parcial. A los 6 años inicia tratamiento con fórmula de NAG una vez al día con buena respuesta y tolerancia desde las 2 semanas de uso, por lo cual mantiene su uso hasta la fecha.
Paciente $N^{\circ}$ 3: Prescolar masculino de 5 años, con diagnóstico de IRLX no sindrómica. De sus antecedentes, destaca hermano con IRLX sindrómica (paciente $\mathrm{N}^{\circ} 2$ ). Desde los 4 años inicia tratamiento con fórmula de NAC aplicada una vez al día con buena respuesta y tolerancia desde las 2 semanas de uso.

Paciente $\mathrm{N}^{\circ} 4$ : Lactante masculino de 1 año 8 meses, con diagnóstico de IRLX no sindrómica. Respecto a sus antecedentes perinatales, nace como bebe colodión de 38 semanas de gestación y parto prolongado. Al mes de vida evoluciona con escamas oscuras y adherentes en tronco y extremidades, respetando cara, pliegues, palmas y plantas. Recibió tratamiento con emolientes y desde los 6 meses de vida, inicia tratamiento con fórmula de NAC aplicada cada 12 horas con buena respuesta y tolerancia desde las 2 semanas de uso. (Figura 2)

Paciente N5: Mujer de 18 años, con diagnóstico de Eritrodermia ictiosiforme congénita (EIC) y estudio genético compatible (mutación CYP4F22). De sus antecedentes perinatales, destaca eritrodermia desde el nacimiento. Estuvo en tratamiento con emolientes y uso restringido de queratolíticos por mala tolerancia. A los 17 años inicia tratamiento con fórmula de NAC cada 12 horas, pero evoluciona con ardor e irritación por lo cual se suspende.

Paciente $\mathrm{N}^{\circ}$ 6: Prescolar femenina de 4 años, con diagnóstico de Ictiosis epidermolítica (IE) y estudio genético compatible (mutación KRT10). Nace con eritrodermia, ampollas y erosiones. Estuvo en tratamiento con emolientes y agentes antisépticos por periodos acotados. A los 3 años inicia tratamiento con fórmula de NAC cada 12 horas, evolucionando con ardor y escasa respuesta, por lo cual se suspende luego de 3 meses.

\section{Discusión}

Si bien las ictiosis hereditarias son patologías poco frecuentes, afectan sustancialmente la calidad de vida de los pacientes y sus familias.

Actualmente solo disponemos de tratamientos sintomáticos, cuyo objetivo es alcanzar un óptimo grado de hidratación, lubricación y queratolisis en las áreas afectadas. En este contexto, los emolientes y queratolíticos como el ácido retinoico, ácido láctico, urea y calcipotriol suelen ser la primera línea de tratamiento, aunque en formas graves son insuficientes. 


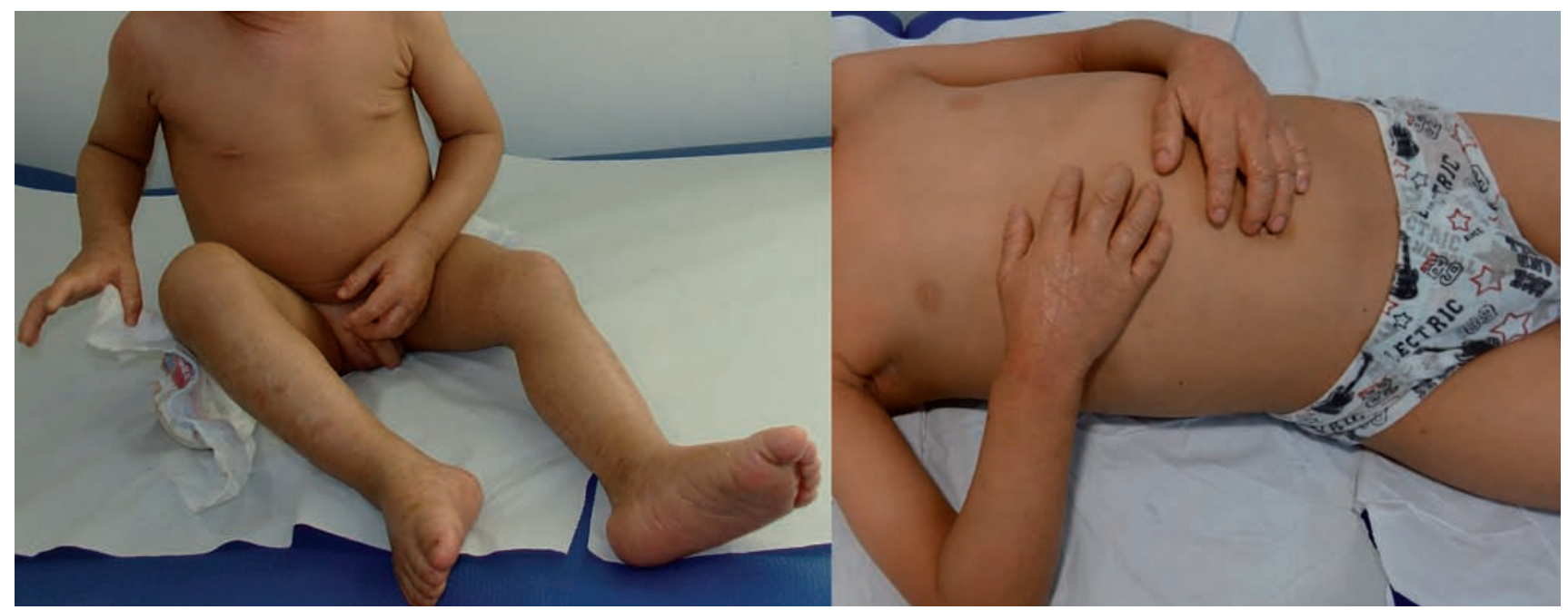

\section{Figura 1}

Paciente $\mathcal{N}^{\circ} 1$ con diagnóstico de Ictiosis lamelar, antes (izquierda) y después (derecha) del tratamiento con fórmula de $\mathcal{N A C}$

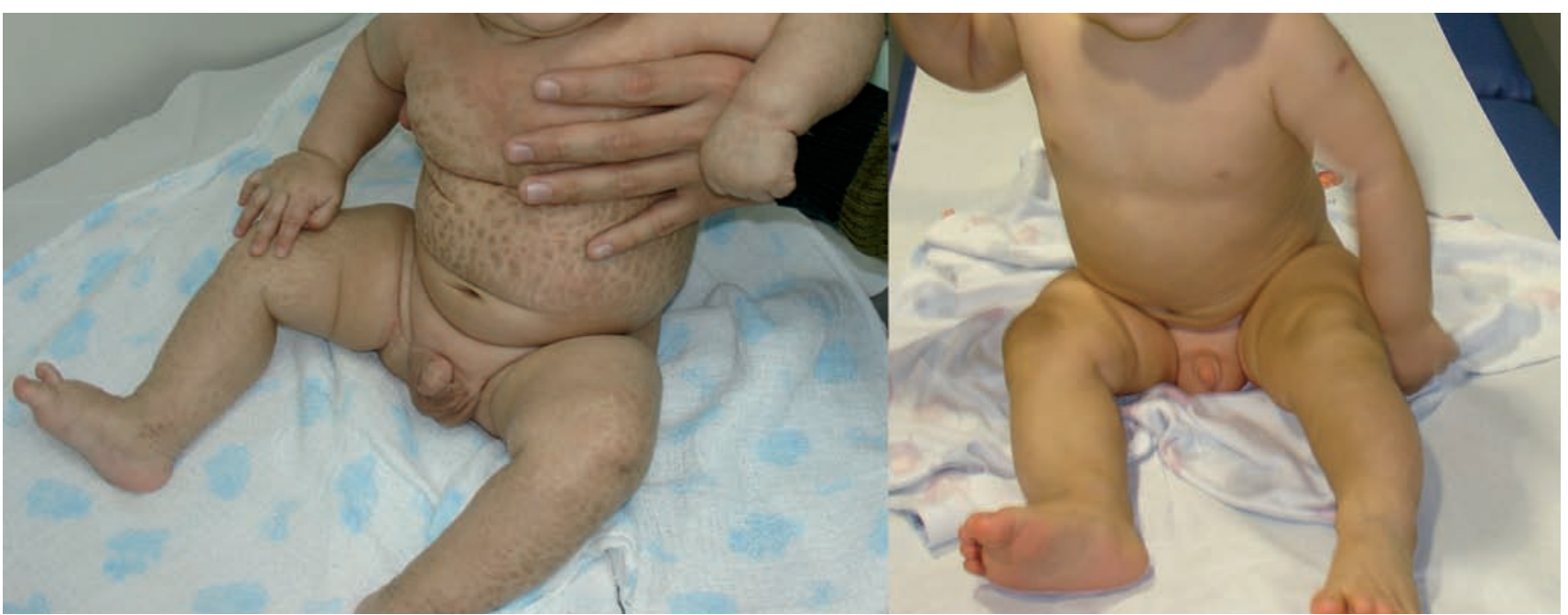

\section{Figura 2}

Paciente $\mathcal{N}^{\circ} 2$ con diagnóstico de Ictiosis recesiva ligada a $X$, antes (izquierda) y después (derecha) del tratamiento con fórmula de $\mathcal{N A C}$

Tabla 1. Descripción de pacientes, tratamiento y evolución

\begin{tabular}{llllll}
\hline Paciente & Sexo/Edad & Tipo de Ictiosis & $\begin{array}{l}\text { Duración de } \\
\text { Tratamiento }\end{array}$ & $\begin{array}{l}\text { Efectos } \\
\text { Adversos }\end{array}$ & Respuesta \\
\hline 1 & M / 7 años & IL & 3 años & - & Satisfactoria \\
2 & M / 7 años & IRLX & 1 año & - & Satisfactoria \\
3 & M / 5 años & IRLX & 1 año & - & Satisfactoria \\
4 & M / 1 años 8 meses & IRLX & 1 año 2 meses & - & Satisfactoria \\
5 & F / 18 años & EIC & 1 año & Ardor & Insatisfactoria \\
6 & F / 4 años & IE & 3 meses & Insatisfactoria
\end{tabular}


Los retinoides sintéticos como isotretinoína o acitretin, actúan regulando la queratinización, pero debido a sus importantes efectos adversos a largo plazo, suelen estar reservados para casos severos o refractarios al tratamiento tópico. ${ }^{3,6}$

La N-Acetilcisteína (NAC) corresponde a un derivado del aminoácido L-cisteína, utilizado tradicionalmente como un agente antioxidante, mucolítico y nefroprotector. ${ }^{7}$ Últimamente, se ha reportado que su uso tópico es efectivo en pacientes con ictiosis lamelar debido a sus efectos anti proliferativos, el cual estaría mediado por un bloqueo reversible del ciclo celular en fase G1, sin efectos citotóxicos. ${ }^{7}$ Es un producto hipoalergénico y con escasos efectos adversos, dentro de los cuales se mencionan irritación, prurito, ardor y mal olor. Este último, es causado por la progresiva oxidación de la NAG una vez aplicada en la piel, liberando componentes que contienen sulfuro. Esto con frecuencia limita su uso y adherencia por parte de los pacientes. Algunos autores han sugerido agregar esencias de fragancias como aceite de romero al 1,5\% para disminuir el mal olor y con ello mejorar la adherencia. ${ }^{8}$

La mezcla de NAC $10 \%$ y urea $5 \%$ ha mostrado sinergia, ya que aparentemente la urea permite un efecto farmacológico óptimo y una mejor penetración transcutánea de los ingredientes activos. ${ }^{4,5,7}$

En este trabajo, se evaluaron los efectos de la fórmula de NAG en diferentes tipos de ictiosis, mostrando un buen perfil de seguridad y eficacia en Ictiosis Lamelar e Ictiosis recesiva ligada a $\mathrm{X}$, sin embargo, en los casos de Eritrodermia ictiosiforme congénita e Ictiosis epidermolítica su uso estuvo restringido por la aparición de ardor e irritación.

Podemos observar con estos resultados que la fórmula de NAC es útil no solo en Ictiosis Lamelar, sino que también en Ictiosis recesiva ligada a X, lo cual no habría sido descrito anteriormente.

\section{Conclusión}

El tratamiento con NAC $10 \%$ y urea $5 \%$ en novobase II presenta buena respuesta y tolerancia en pacientes con IL e IRLX, por lo que podría ser considerado en el tratamiento habitual de estas patologías. Por el contrario, ambos pacientes con IE y EIC presentaron mala tolerancia y su utilización en estos casos podría desesti- marse. Aun cuando resulta necesario un mayor número de pacientes para confirmar los datos obtenidos, nuestro estudio constituye una buena aproximación para orientar su tratamiento, considerando la baja prevalencia de la enfermedad y los escasos reportes de casos tratados con esta fórmula en la literatura.

\section{REFERENCIAS BIBLIOGRÁFICAS}

1. Oji V, Tadini G, Akiyama M, et al. Revised nomenclature and classification of inherited ichthyoses: Results of the First Ichthyosis Consensus Conference in Sorèze 2009. J Am Acad Dermatol 2010; 63:607- 41

2. Rodríguez-Pazos L, Ginarte M, Vega A, Toribio J. Clasificación clínica y molecular de las ictiosis. Piel (Barc) 2012; 27:314-26

3. Rodríguez-Pazos L, Ginarte M, Vega A, Toribio J. Ictiosis congénitas autosómicas recesivas. Actas Dermosifiliogr 2013; 104(4):270-284

4. Bassotti A, Moreno S, Criado E. Successful treatment with topical $\mathrm{N}$-acetylcysteine in urea in five children with congenital lamellar ichthyosis. Pediatr Dermatol 2011; 28: 451-455

5. Redondo P, Bauzá A. Topical N-acetylcysteine for lamellar ichthyosis. Lancet 1999; 354:1880

6. Aranegui B, Hernández-Martín Á. Tratamiento de las ictiosis congénitas. Piel (Barc) 2013; 29(1):43-48

7. Sarici SÜ, Sahin M, Yurdakök M. Topical N-acetylcysteine treatment in neonatal ichthyosis. Turk J Pediatr 2003; 45:245-247

8. Davila-Seijo P, Flórez A, Davila-Pousa G, No N, Ferreira G, De la Torre C. Topical N-acetylcysteine for the treatment of lamellar ichthyosis: an improved formula. Dermatol 2014; 31(3):395-7 